\title{
Is Cherenkov luminescence bright enough for photodynamic therapy?
}

To the Editor - It has recently been proposed that Cherenkov luminescence from radionuclides can be used to activate tumour-localized photosensitizing nanoparticles $\left(\mathrm{TiO}_{2}\right)$ and cause enhanced tumour cell death ${ }^{1}$. This approach is proposed as a new method for circumventing the depth penetration limits of conventional light-based therapies. While the results of this study leave little doubt that radionuclides and photosensitizers interact in a synergistic manner, they do not prove that this interaction occurs through Cherenkov luminescence.

Cherenkov luminescence is known to be an exceedingly dim phenomenon ${ }^{2}$. Average Cherenkov emission from ${ }^{18} \mathrm{~F}$ is approximately three photons per radioactive decay in water (refractive index $n=1.33$ ) over the $250-800 \mathrm{~nm}$ range $\mathrm{e}^{3}$. In terms of energy, Cherenkov light represents less than $0.006 \%$ of the total energy released during the radioactive decay of ${ }^{18} \mathrm{~F}$. The vast majority of the energy (>99.99\%) is dissipated through molecular excitation, ionization, Bremsstrahlung radiation and heat.

On average, a single ${ }^{18} \mathrm{~F}$ decay $(250 \mathrm{keV})$ yields 6,800 hydroxyl radicals $\left(\mathrm{OH}^{\bullet}\right)$ through radiolysis of water ${ }^{4}$. These $\mathrm{OH}^{\bullet}$ radicals play a major role in mediating the biological action of ionizing radiation on DNA. For Cherenkov luminescence to produce a significant biological effect, its action on DNA must be comparable to or greater than that of $6,800 \mathrm{OH}^{\bullet}$ radicals. However, due to the bandgap of $\mathrm{TiO}_{2}(3.2 \mathrm{eV})$, it is impossible for three Cherenkov photons to produce more than three $\mathrm{OH}^{\bullet}$ radicals.

An alternative explanation for the observed increase in free radical production and cell death is that ionizing positrons interact directly with $\mathrm{TiO}_{2}$ nanoparticles to enhance cellular toxicity, without intervention of Cherenkov light. One simple way to test this alternative mechanism would be to irradiate the specimens with X-rays at energies below the Cherenkov threshold, with and without photosensitizer. It is also worth noting that a previous study reporting activation of $\mathrm{TiO}_{2}$ nanoparticles from radioactive ${ }^{32} \mathrm{P}$ did not invoke Cherenkov luminescence as the mechanism of action ${ }^{5}$.

\section{Guillem Pratx ${ }^{\star}$ and Daniel S. Kapp}

Department of Radiation Oncology, Stanford

University School of Medicine, Stanford, CA, USA.

*e-mail:pratx@stanford.edu

Published online: 4 May 2018

https://doi.org/10.1038/s41565-018-0142-y

References

1. Kotagiri, N. et al. Nat. Nanotech. 10, 370-379 (2015)

2. Glaser, A. K. et al. Phys. Med. Biol. 60, 6701-6718 (2015).

3. Gill, R. K., Mitchell, G. S. \& Cherry, S. R. Phys. Med. Biol. 60, 4263-4280 (2015).

4. Schwarz, H. A. J. Chem. Educ. 58, 101-105 (1981)

5. Rajh, T., Dimitrijevic, N. M., Elhofy, A. \& Rozhkova, E. in Handbook of Nanophysics (ed. Sattler, K. D.) 1-28 (CRC Press, Boca Raton, 2010).

\section{Reply to 'Is Cherenkov luminescence bright enough for photodynamic therapy?'}

\begin{abstract}
Kotagiri, Laforest and Achilefu reply The interaction of beta radionuclides with semiconductors and photocatalysts such as $\mathrm{TiO}_{2}$ remains an underexplored and exciting area of investigation. These interactions could potentially involve many processes, including: (1) radiolysis of bulk water by ionizing radiation, generating electrons and hydroxyl radicals; (2) generation of electron and hole pairs in $\mathrm{TiO}_{2}$ through radiolytic energy conversion ${ }^{1}$; and (3) generation of electron and hole pairs in $\mathrm{TiO}_{2}$ through Cherenkov luminescence energy transfer and other luminescence-generating phenomena. The various types of energy emission from a decaying radionuclide are complex and diverse. While it would be immensely important and interesting to explore the various phenomena dictating material behaviour on interaction with these sources, we took steps to initially demonstrate the factors that do not play a singular role in the effects we observed.
\end{abstract}

To minimize the contribution of pathway (1) above, we used an ${ }^{18} \mathrm{~F}$-fludeoxyglucose $\left({ }^{18} \mathrm{~F}-\mathrm{FDG}\right)$ dose that ensured ${ }^{18} \mathrm{~F}$ radiolysis did not result in any observable biological effect in the absence of $\mathrm{TiO}_{2}$. Using a HT1080 tumour model, a reduction in tumour burden was not observed up to $30 \mathrm{MBq}$. In pathways (2) and (3) above, hydrogen peroxide, singlet oxygen, hydroxyl and superoxide radicals are produced on the solid-liquid interface through catalysis ${ }^{2,3}$. The cumulative effects of these radicals is expected to induce cytotoxic effects in tumours. Superoxide dismutase can convert superoxide radicals to hydrogen peroxide and the increased iron content in tumour cells facilitates the conversion of hydrogen peroxide to hydroxyl radicals. Hydrogen peroxide is sufficiently stable to traverse cells before conversion to the reactive hydroxyl radicals. Though hydroxyl radicals have extremely short lifespan and path lengths, their high reactivity is known to induce localized cytotoxicity ${ }^{4}$.

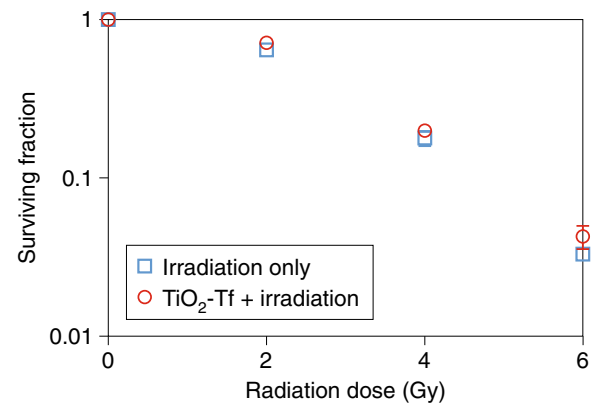

Fig. 1 | Clonogenic assay comparing the effect of $<250 \mathrm{keV}$ X-ray irradiation on HT1080 cancer cells with and without $\mathrm{TiO}_{2}$ nanoparticles $\left(2.5 \mu \mathrm{g} \mathrm{ml}^{-1}\right)$. Values represent mean \pm standard error of the mean. Tf, transferrin.

Beta particles have short path lengths ( $100 \mu \mathrm{m}$ to $5 \mathrm{~mm}$, depending on the radionuclide) compared to Cherenkov light, which has a wider sphere of influence and therefore can have a significant volume 